Check for updates

Cite this: Chem. Commun., 2017, 53,4922

Received 3rd March 2017, Accepted 7th April 2017

DOI: 10.1039/c7cc01671a

rsc.li/chemcomm

\section{Conjunctive functionalization of vinyl boronate complexes with electrophiles: a diastereoselective three-component coupling $\dagger$}

\author{
Roly J. Armstrong, (D) Christopher Sandford, Cristina García-Ruiz and \\ Varinder K. Aggarwal (D) *
}

\begin{abstract}
A method for the conjunctive functionalization of vinyl boronate complexes with electrophiles is described. The overall process represents a three-component coupling between a vinyl boronic ester, carbon nucleophile and an electrophile, thus affording complex multifunctionalized products from simple starting materials. The diastereoselectivity (syn or anti) of this process is strongly dependent upon the nature of the electrophile.
\end{abstract}

Boronic esters are versatile reagents which have been employed in a wide array of synthetic transformations. ${ }^{1}$ They are easy to handle, bench stable materials that can be readily activated by the addition of a suitable nucleophile to form a boronate complex. ${ }^{2}$ It is well known that the trivalent boron atom of allylic boronic esters can undergo activation with a Lewis base, leading to strongly nucleophilic character at the $\gamma$-position. Consequently, allylic boronic esters can be combined with a range of electrophiles, most notably in allylborations of aldehydes and imines (Scheme 1a). ${ }^{3}$ In contrast, the reactivity of boronate complexes derived from vinyl boronic esters has been less extensively explored. Upon treatment of a vinyl boronate complex with an electrophile two possible reaction pathways could be envisaged: (1) direct functionalization of an electron rich carbon-boron bond; ${ }^{4}(2)$ a conjunctive functionalization process, in which the $\pi$-system of the vinyl group reacts with the electrophile, triggering a 1,2-metallate rearrangement (Scheme 1a). The latter class of reaction is of particular interest since it represents a three-component coupling between a nucleophile, a vinyl boronic ester and an electrophile. Moreover, the boronic ester is retained in the product, serving as a versatile handle for further functionalization., ${ }^{1,5}$

Although the electrophilic functionalization of vinyl boronate complexes has not received a great deal of attention, several interesting examples have been reported. ${ }^{6-8}$ Recently, Morken

School of Chemistry, University of Bristol, Cantock's Close, Bristol BS8 1TS, UK. E-mail:v.aggarwal@bristol.ac.uk

$\dagger$ Electronic supplementary information (ESI) available. CCDC 1534237. For ESI and crystallographic data in CIF or other electronic format see DOI: 10.1039/c7cc01671a (a) Nucleophilic functionalization of allyl and vinyl boronate complexes

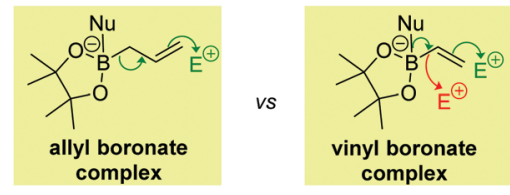

(b) Conjunctive cross-coupling (Morken)

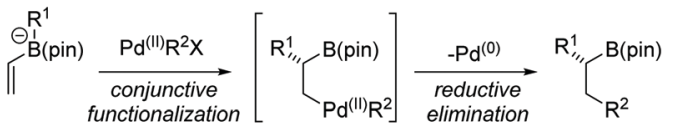

(c) Zweifel Olefination (Evans, Brown, Negishi)

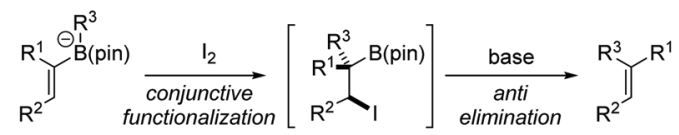

(d) Syn-selective Olefination (Aggarwal)

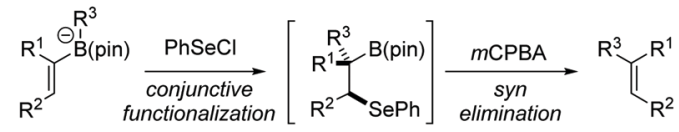

(e) This work

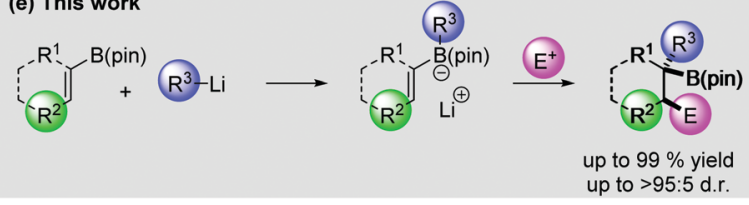

Scheme 1 Previous work and strategy for three-component conjunctive coupling.

and co-workers disclosed an elegant enantioselective conjunctive variant of the Suzuki-Miyaura reaction (Scheme 1b). ${ }^{9}$ Mechanistically, this process involves $\beta$-functionalization of a vinyl boronate complex by a palladium(II) aryl species, followed by reductive elimination. Another notable example of conjunctive functionalization is the Zweifel olefination (Scheme 1c). ${ }^{10}$ In this process, a vinyl boronate complex undergoes stereospecific reaction with iodine, resulting in the formation of a $\beta$-iodoboronic ester (likely via a transient iodonium ion). This intermediate is unstable, undergoing anti elimination in the presence of a weak base to afford olefin products. Recently we reported a conjunctive functionalization of 
vinyl boronate complexes with $\mathrm{PhSeCl}$ (Scheme 1d). ${ }^{11}$ The $\beta$-selenoboronic ester intermediates were not isolated, but were treated with mCPBA, resulting in syn elimination to form the corresponding alkenes. In this work, we aimed to develop a conjunctive threecomponent coupling process to obtain boron containing products, which would not undergo $\beta$-elimination. The scope with respect to each of the three coupling partners has been evaluated and the factors that influence the efficiency and diastereoselectivity of these reactions are discussed.

We commenced our study by evaluating the functionalization of $E$-disubstituted boronic ester 1. Addition of PhLi to a solution of 1 in THF at $-78{ }^{\circ} \mathrm{C}$ resulted in clean boronate complex formation and upon addition of $\mathrm{PhSeCl}$, we were pleased to find that the product of three-component coupling 2 could be isolated in $82 \%$ yield as a single diastereoisomer (Table 1). The anti diastereoselectivity of the reaction was confirmed by X-ray crystallographic analysis of the product. We then carried out an identical sequence beginning with $Z$-vinyl boronic ester 3 . This provided the syn diastereoisomer 4 (>95:5 d.r.) in 70\% yield, indicating that the process is completely stereospecific. Primary and secondary organolithiums could also be employed in the process, affording alkyl-substituted products $\mathbf{5}$ and $\mathbf{6}$ in excellent yields and very high diastereoselectivity. MeLi also served as a successful nucleophile, affording 7 in $79 \%$ yield, which is particularly noteworthy given the known poor migratory aptitude of a methyl group. ${ }^{10 c} \mathrm{~A}$ vinyl boronic ester containing an $\alpha$-substituent was also well tolerated and $\mathbf{9}$ was formed in $78 \%$ yield. When trisubstituted vinyl boronic ester $\mathbf{1 0}$ was used as the starting material, 11 was obtained in $93 \%$ yield ( $>95: 5$ d.r.). The other diastereoisomer could be obtained in $93 \%$ yield by employing the corresponding $Z$-vinyl boronate $\mathbf{1 2}$ as the starting material. Homobenzyl substituted boronic esters 14 and 16 also reacted successfully, affording products $\mathbf{1 5}$ and $\mathbf{1 7}$ in excellent yields and diastereoselectivity. A silyl-ether-containing substrate also reacted cleanly, affording 19 in $73 \%$ yield. We were pleased to find that cyclic boronic esters $\mathbf{2 0}$ and $\mathbf{2 2}$ also efficiently underwent the desired reaction. The latter example demonstrates that high chemoselectivity can be obtained in the presence of a Boc-protected amine.

We next attempted the coupling of $\beta, \beta$-disubstituted vinyl boronic ester 24. Disappointingly, we found that the desired product 25 was formed in a modest yield of $34 \%$ yield, along with $45 \%$ yield of vinyl selenide 26 . A plausible mechanism accounting for the formation of $\mathbf{2 6}$ is outlined in Scheme 2. Initially, the vinyl boronate complex derived from $\mathbf{2 4}$ undergoes reaction with $\mathrm{PhSeCl}$ to form a zwitterionic seleniranium intermediate. This species can either undergo the desired 1,2-migration, or eliminate to form vinyl selenide 26 along with $\mathrm{PhB}(\mathrm{pin}){ }^{12}$ For the majority of examples, the former pathway predominates, but in the case of boronate complexes derived from $\beta, \beta$-disubstituted boronic esters, the ability to build up partial positive charge at the tertiary $\beta$-carbon allows the $\mathrm{C}_{\beta}-\mathrm{Se}$ bond to lengthen and facilitates the elimination process. We found that the reaction solvent has a significant influence on which pathway is preferred (see ESI $\dagger$ for full details of optimization). Ultimately, we found that in a $3: 1 \mathrm{THF} / \mathrm{DMF}$ mixture, formation of $\mathbf{2 6}$ was suppressed and 25 could be obtained as a single diastereomer in $61 \%$ yield.
Table 1 Conjunctive three-component coupling: boronic ester and RLi scope $^{a}$

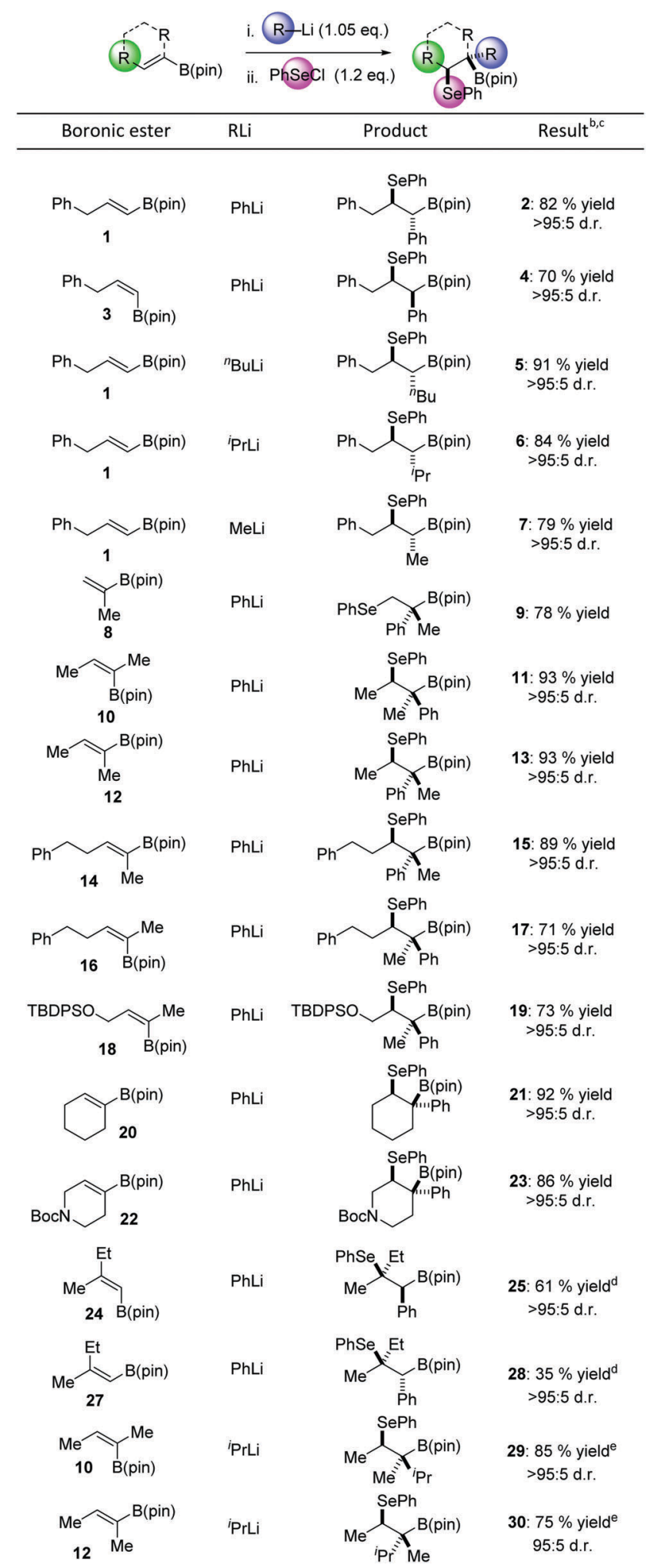

${ }^{a}$ Reaction conditions: vinyl boronic ester (1.0 eq.), RLi (1.05 eq.), THF; then $\mathrm{PhSeCl}$ (1.2 eq.), THF. ${ }^{b}$ Yields of isolated products. ${ }^{c}$ d.r. was determined by integration of crude ${ }^{1} \mathrm{H}$ NMR spectra. ${ }^{d}$ Reaction with $\mathrm{PhSeCl}$ carried out in $3: 1 \mathrm{THF} / \mathrm{DMF} .{ }^{e}$ Reaction with $\mathrm{PhSeCl}$ carried out in $1: 1 \mathrm{THF} / \mathrm{CF}_{3} \mathrm{CH}_{2} \mathrm{OH}$. 


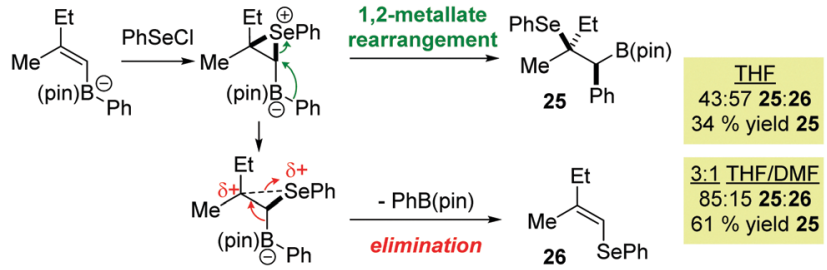

Scheme 2 Competing pathways in the formation of $\mathbf{2 5}$ and the effect of solvent.

These conditions were also applied to the three-component coupling of isomeric vinyl boronic ester $\mathbf{2 7}$ affording the product 28 in $35 \%$ yield as a single diastereomer (Table 1).

We next carried out a three-component coupling between 10, ${ }^{\mathrm{i}} \mathrm{PrLi}$ and $\mathrm{PhSeCl}$. Given that this vinyl boronic ester had been reacted with an aryl lithium to afford $\mathbf{1 1}$ in very high diastereoselectivity (vide supra) we did not anticipate that this process would be problematic. We were therefore surprised to find that 29 was isolated as an 88:12 mixture of diastereoisomers (Scheme 3). We attribute this reduced diastereoselectivity to the pathway shown in Scheme 3, whereby partial opening of the seleniranium intermediate enables a partial positive charge to develop at the tertiary $\alpha$-carbon. This allows a syn 1,2-migration process to compete with the usual anti migration, resulting in the formation of both 29 and 30. After solvent optimization (see ESI $\dagger$ for full details) we found that carrying out the reaction in a 1:1 mixture of THF/trifluoroethanol suppressed the undesired pathway, providing 29 in very high diastereoselectivity. We suspect that trifluoroethanol is able to modulate the reactivity of the intermediate seleniranium cation by functioning as a hydrogen bond donor to the basic pinacol oxygen atoms. ${ }^{13}$ An analogous reaction with isomeric vinyl boronic ester 12 afforded the product 30 in $75 \%$ yield and $95: 5$ d.r.

Taking boronate complexes derived from isomeric vinyl boronic esters 10 and $\mathbf{1 2}$ as representative examples, coupling with a series of electrophiles was investigated (Table 2; results with $\mathrm{PhSeCl}$ are also shown for clarity). With $\mathrm{PhSCl}$ the reaction was extremely efficient, producing $\mathbf{3 1}$ and $\mathbf{3 2}$ in excellent yield. Both products were obtained as a single diastereoisomer indicating that the process is stereospecific. To confirm the relative configuration of $\mathbf{3 1}$ and $\mathbf{3 2}$, we subjected them to methoxide promoted anti elimination. As expected, this resulted in the formation of isomeric alkenes $\mathbf{3 9}$ and $\mathbf{4 0}$, respectively (Table 3 , entries 3 and 4). We next investigated fluorination of vinyl boronate complexes. ${ }^{2 d}$ Employing Selectfluor, $\beta$-fluoroboronic

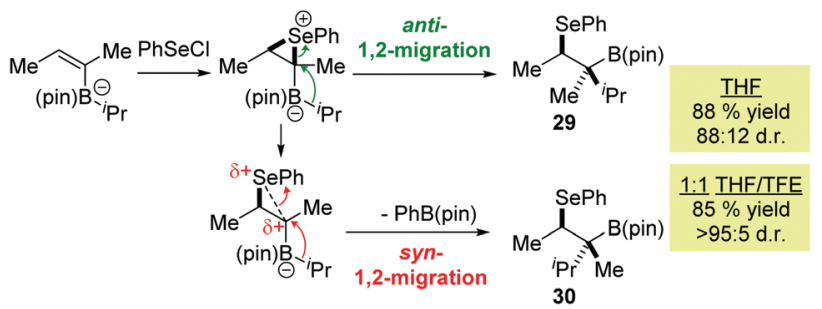

Scheme 3 Eroded diastereoselectivity in the formation of 29. TFE = $\mathrm{CF}_{3} \mathrm{CH}_{2} \mathrm{OH}$.
Table 2 Conjunctive three-component coupling: electrophile scope ${ }^{a}$

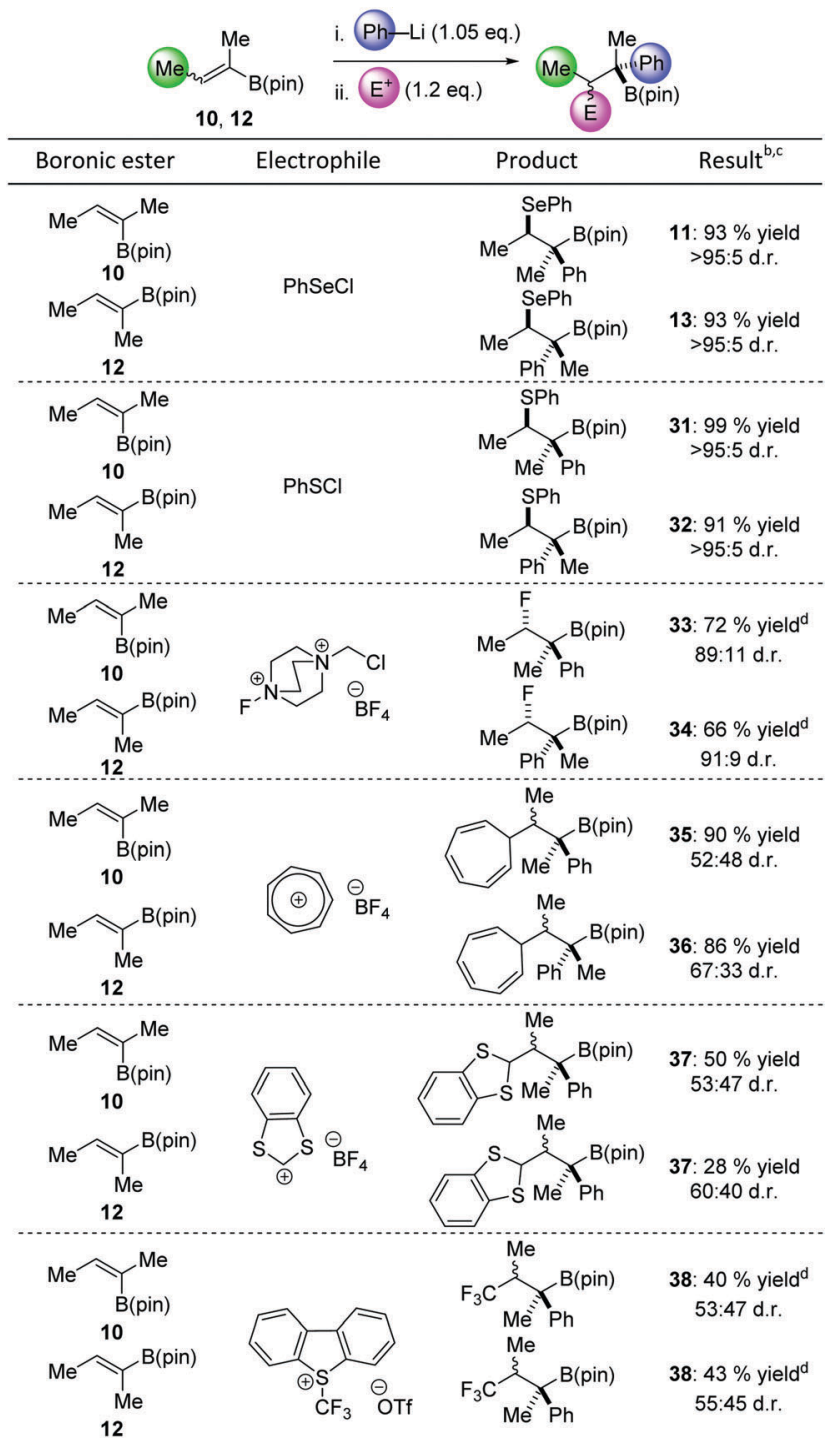

${ }^{a}$ Reaction conditions: vinyl boronic ester (1.0 eq.), PhLi (1.05 eq.); then electrophile (1.2 eq.). For details of solvent and reaction conditions see ESI. ${ }^{b}$ Yields of isolated products. ${ }^{c}$ d.r. was determined by integration of crude ${ }^{1} \mathrm{H}$ and ${ }^{19} \mathrm{~F}$ NMR spectra. ${ }^{d}{ }^{19} \mathrm{~F}$ NMR yield using $\alpha, \alpha, \alpha$-trifluorotoluene as an internal standard.

esters 33 and 34 were obtained in good yield and high diastereoselectivity (Table 2). Upon exposure of these compounds to sodium methoxide, we were surprised to isolate the unexpected isomers of alkenes $\mathbf{4 0}$ and $\mathbf{3 9}$ (Table 3, entries 5 and 6). There are two possible explanations for this observation: (1) $\beta$-fluoroboronic esters $\mathbf{3 3}$ and $\mathbf{3 4}$ are formed by a selective syn migration process; (2) elimination proceeds via a syn pathway. To differentiate between these two scenarios, we carried out fluorination of a cyclic boronate complex, obtaining $\mathbf{4 1}$ as a single diastereoisomer (Scheme 4). HOESY experiments revealed that an unusual syn migration process had occurred. By analogy, we propose that a similar syn pathway is involved in the formation of $\mathbf{3 3}$ and $\mathbf{3 4}$. This surprising result suggests that anti diastereoselectivity is only observed in cases where vinyl boronate complexes are activated 
Table 3 Determination of relative configuration by anti elimination ${ }^{a}$

\begin{tabular}{|c|c|c|c|c|}
\hline & $\mathrm{Me} \overbrace{\mathrm{Me}}^{\mathrm{B}(\mathrm{phin})}$ & $\underset{\mathrm{THF} / \mathrm{MeOH}}{\stackrel{\mathrm{NaOMe}}{\longrightarrow}}$ & $\mathrm{Me} \overbrace{\substack{\mathrm{Me} \\
39}}^{\mathrm{Ph}}$ & $\underbrace{\mathrm{Ph}}_{P 0}$ \\
\hline Entry & Substrate & d.r. & Major product & $E / Z$ selectivity ${ }^{b}$ \\
\hline 1 & $11(E=S e P h)$ & $>95: 5$ & 39 & $>95: 5$ \\
\hline 2 & $13(\mathrm{E}=\mathrm{SePh})$ & $>95: 5$ & 40 & $<5: 95$ \\
\hline 3 & $31(\mathrm{E}=\mathrm{SPh})$ & $>95: 5$ & 39 & $96: 4$ \\
\hline 4 & $32(\mathrm{E}=\mathrm{SPh})$ & $>95: 5$ & 40 & $29: 71$ \\
\hline 5 & $33(\mathrm{E}=\mathrm{F})$ & $89: 11$ & 40 & $17: 83$ \\
\hline 6 & $34(\mathrm{E}=\mathrm{F})$ & $91: 9$ & 39 & $92: 8$ \\
\hline
\end{tabular}

${ }^{a}$ Reaction conditions: $\beta$-substituted boronic ester (1.0 eq.), NaOMe (10 eq.), THF/MeOH $(8: 1) .{ }^{b}$ Determined by integration of the crude ${ }^{1} \mathrm{H}$ NMR spectrum.

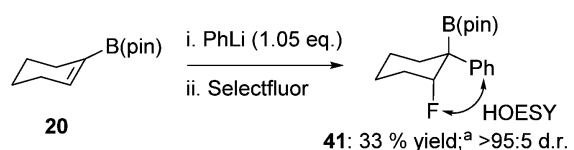

Scheme 4 Probing the diastereoselectivity of fluorination. Reaction conditions: vinyl boronic ester (1.0 eq.), PhLi (1.05 eq.), THF; then Selectfluor I (1.2 eq.), MeCN/THF $(5: 1) .{ }^{\text {a }}{ }^{19} \mathrm{~F}$ NMR yield using $\alpha, \alpha, \alpha$-trifluorotoluene as an internal standard.

by electrophiles that form closed three-membered ring intermediates. In line with this hypothesis, electrophilic carbon reagents, such as tropylium tetrafluoroborate and 1,3-benzodithiolylium tetrafluoroborate led to competing syn and anti functionalization, resulting in low levels of diastereoselectivity (Table 2). Finally, we investigated the reaction of vinyl boronate complexes with Umemoto's reagent, a process that is expected to occur by addition of a trifluoromethyl radical followed by single-electron oxidation to form a carbocation intermediate. ${ }^{14}$ As expected, near identical diastereomeric mixtures of trifluoromethylated products were obtained regardless of which isomer of vinyl boronic ester was employed.

In summary, a conjunctive three-component coupling between vinyl boronic esters, carbon nucleophiles and electrophiles has been developed. The diastereoselectivity of the process is strongly dependent upon the nature of the electrophile. Reactions that proceed via closed three-membered cyclic intermediates exhibit very high anti diastereoselectivity. In the case of more reactive, charged electrophiles, the increasingly asynchronous nature of the bond formation enables syn migration to compete, in some cases becoming the preferred pathway. This work will be of use for the synthesis of boron-containing materials and will inform the development of other conjunctive functionalization processes.

We thank EPSRC (EP/I038071/1) and Bristol University for financial support. C. G.-R. thanks the Ramón Areces Foundation for a postdoctoral fellowship. We thank Dr Eddie Myers for helpful discussions, Dr Hazel A. Sparkes and Dr Natalie E. Pridmore for assistance with X-ray crystallography and Dr E. Kriemen for carrying out preliminary studies.

\section{Notes and references}

1 Synthesis and application of organoboron compounds, ed. E. Fernández and A. Whiting, Springer, Heidelberg, 2015.

2 (a) H. C. Brown, N. R. De Lue, G. W. Kabalka and H. C. Hedgecock, J. Am. Chem. Soc., 1976, 98, 1290-1291; (b) H. C. Brown and B. Singaram, Acc. Chem. Res., 1988, 21, 287-293; (c) R. LaroucheGauthier, T. G. Elford and V. K. Aggarwal, J. Am. Chem. Soc., 2011, 133, 16794-16797; (d) C. Sandford, R. Rasappan and V. K. Aggarwal, J. Am. Chem. Soc., 2015, 137, 10100-10103.

3 (a) Organic Reactions: Allylboration of Carbonyl Compounds, ed. H. Lachance and D. G. Hall, Wiley, Weinheim, 2009, vol. 73; (b) J. W. J. Kennedy and D. G. Hall, Angew. Chem., Int. Ed., 2003, 42, 4732-4739; (c) S. E. Denmark and J. Fu, Chem. Rev., 2003, 103, 2763-2794.

4 (a) N. R. Candeias, F. Montalbano, P. M. S. D. Cal and P. M. P. Gois, Chem. Rev., 2010, 110, 6169-6193; (b) A. J. J. Lennox and G. C. LloydJones, Chem. Soc. Rev., 2014, 43, 412-443; (c) H. C. Brown, T. Hamaoka and N. Ravindran, J. Am. Chem. Soc., 1973, 95, 5786-5788; (d) S. Lee and D. W. C. MacMillan, J. Am. Chem. Soc., 2007, 129, 15438-15439; (e) A. Mitchell and J. W. Bode, J. Am. Chem. Soc., 2009, 131, 18057-18059.

5 (a) H. K. Scott and V. K. Aggarwal, Chem. - Eur. J., 2011, 17, 13124-13132; (b) R. Jana, T. P. Pathak and M. S. Sigman, Chem. Rev., 2011, 111, 1417-1492; (c) C.-Y. Wang, J. Derosa and M. R. Biscoe, Chem. Sci., 2015, 6, 5105-5113; (d) C. Sandford and V. K. Aggarwal, Chem. Commun., 2017, 53, DOI: 10.1039/C7CC01254C. 6 For selected examples with alkynyl boronate complexes, see: (a) M. Naruse, K. Utimoto and H. Nozaki, Tetrahedron Lett., 1973, 14, 2741-2744; (b) N. Miyaura, T. Yoshinari, M. Itoh and A. Suzuki, Tetrahedron Lett., 1974, 15, 2961-2964; (c) K. K. Wang and S. Dhumrongvaraporn, Tetrahedron Lett., 1987, 28, 1007-1010; (d) N. Ishida, W. Ikemoto, M. Narumi and M. Murakami, Org. Lett., 2011, 13, 3008-3011.

7 For selected examples with aromatic boronate complexes, see: (a) G. M. Davies, P. S. Davies, W. E. Paget and J. M. Wardleworth, Tetrahedron Lett., 1976, 17, 795-798; (b) I. Akimoto and A. Suzuki, Synthesis, 1979, 146-147; (c) M. Ishikura and H. Kato, Tetrahedron, 2002, 58, 9827-9838; (d) A. Bonet, M. Odachowski, D. Leonori, S. Essafi and V. K. Aggarwal, Nat. Chem., 2014, 6, 584-589; (e) M. Odachowski, A. Bonet, S. Essafi, P. Conti-Ramsden, J. N. Harvey, D. Leonori and V. K. Aggarwal, J. Am. Chem. Soc., 2016, 138, 9521-9532.

8 For conjunctive intramolecular alkylation, see: Y. Kobayashi, M. Asano and Y. Kiyotsuka, Heterocycles, 2009, 77, 787-791.

9 (a) L. Zhang, G. J. Lovinger, E. K. Edelstein, A. A. Szymaniak, M. P. Chierchia and J. P. Morken, Science, 2016, 351, 70-74; (b) G. J. Lovinger, M. D. Aparece and J. P. Morken, J. Am. Chem. Soc., 2017, 139, 3153-3160.

10 (a) G. Zweifel, H. Arzoumanian and C. C. Whitney, J. Am. Chem. Soc., 1967, 89, 3652-3653; (b) D. A. Evans, T. C. Crawford, R. C. Thomas and J. A. Walker, J. Org. Chem., 1976, 41, 3947-3953; (c) H. C. Brown and N. G. Bhat, J. Org. Chem., 1988, 53, 6009-6013; (d) S. Xu, C.-T. Lee, H. Rao and E. Negishi, Adv. Synth. Catal., 2011, 353, 2981-2987.

11 R. J. Armstrong, C. García-Ruiz, E. L. Myers and V. K. Aggarwal, Angew. Chem., Int. Ed., 2017, 56, 786-790.

12 In analogy with related reactions of vinyl silanes, this process proceeds with stereochemical retention: T. A. Blumenkopf and L. E. Overman, Chem. Rev., 1986, 86, 857-873.

13 I. Shuklov, N. Dubrovina and A. Börner, Synthesis, 2007, 2925-2943.

14 (a) Y. Wang, A. Noble, C. Sandford and V. K. Aggarwal, Angew. Chem., Int. Ed., 2017, 56, 1810-1814; (b) M. Kischkewitz, K. Okamoto, C. Mück-Lichtenfeld and A. Studer, Science, 2017, 355, 936-938. 